\title{
On the use of discrete - time Markov process for HIVIAIDs epidemic modelling
}

\section{OGUNMOLA ADENIYI OYEWOLE}

Department of Mathematics and Statistics, Faculty of Pure and Natural Science, Federal University Wukari, Taraba State

\section{Email address:}

adeniyiogunmola@gmail.com

\section{To cite this article:}

OGUNMOLA ADENIYI OYEWOLE. On the use of Discrete - Time Markov Process for HIV/AIDs Epidemic Modelling. American Journal of Applied Mathematics. Vol. 2, No. 1, 2014, pp. 21-28. doi: 10.11648/j.ajam.20140201.14

\begin{abstract}
In this research, a discrete-time Markov process for HIV/AIDs epidemic modeling, which takes into account the dynamic of the HIV; the number of susceptible contracting HIV, the number of infective developing AIDS and the parameters influencing these outcomes is designed. This is to determine the behaviour of the epidemic and to keep it under control. Each parameter in the model was varied at different values while others are kept constant to determine the effects of the parameter on the disease states, and to ultimately determine the more important parameter(s) necessary to control the epidemic. By simulation, it was revealed that the susceptible people in a population depletes in a negative exponential form after contracting HIV, the infectives grow and decay in a log logistic form, while the AIDS people in the population grow in a positive exponential form. The rate at which susceptible becomes infective and the rate at which infective becomes AIDS are crucial parameters which when kept low, the epidemic is kept under control.
\end{abstract}

Keywords: Discrete Time, Markov Process, HIV/AIDS, Susceptible, Infective, Models

\section{Introduction}

AIDS is an infectious disease caused by a retrovirus called Human Immunodeficiency Virus (HIV), Coffin (1986). The prime targets of HIV are the $\mathrm{T}_{4}$ cells $\left(\mathrm{CD} 4^{(+)} \mathrm{T}\right.$ helper cells) resulting in the collapse of immunization system in the body and hence death.

In Nigeria, AIDS was first diagnosed in 1985 in a female teenaged less than 14 years but was reported in 1986. This case was diagnosed in Lagos, one of the most populous city in Nigeria Nasidi et al (1986). Twenty-seven years after, the disease has become a massive epidemic which has become not only a health burden but also a socio-economic problem. One of the most urgent challenging tasks is how to control the HIV epidemic. For this purpose and for understanding the HIV epidemic, mathematical models that take into account the dynamics of the HIV epidemic and the HIV biology are definitely needed.

Van Druten et al (1987) and Isham (1988) derived deterministic approximation to susceptible becoming infectives and infectives becoming AIDS in a population of homosexuals. The prevalence of the infection increases exponentially in the early stages of the epidemic. According to Trottier and Philippe (2002), the first step in deterministic model consists of having a complete and realistic picture of the biology of the disease understudy, and select a parsimonious model. This was applied in measles, an infectious disease. It was discovered that any prediction of the number of new cases in an epidemics is not straight forward as the relationships among the various model compartment are non-linear. Allen and Burgin (1998) developed a discrete time deterministic and stochastic Susceptible, Infective, and Susceptible (SIS) models. They also developed a discrete time deterministic and stochastic Susceptible, Infective, and Recovered (SIR) models. These previous works are the framework for the model adopted in this research work to understand the behaviour of the three states of the HIV/AIDS epidemic and determine some crucial parameters to control the epidemic.

The objective of this research is to use the discrete time Markov process for HIV/AIDS epidemic modeling to determine the behaviour of the epidemic on the number of susceptibles, number of infectives and number of AIDS cases. It is also to determine the effects of the parameters influencing the epidemic on the numbers of these three groups to ultimately determine the crucial parameters to control the HIV/AIDS epidemic. The scope of this work is limited to number of susceptibles contracting HIV, number of infectives developing into AIDS, rates susceptible becomes infective, rates infective become AIDS, AIDS 
death rates and natural death rates, and using computer simulation to determine the result of the model.

\section{The Model}

We first describe the deterministic epidemic model. The model consists of four differential equations, one for each of the three disease states; susceptibles, infectives and AIDS cases, with the number in each class denoted by $S(t)$, $\mathrm{I}(\mathrm{t})$ and $\mathrm{A}(\mathrm{t})$ respectively. Secondly, we develop a stochastic model, a discrete - time Markov process model, based on the deterministic model. The stochastic variability in this model is due to the variability in the immigrant, death and infection process and does not account for environmental variability. Thirdly, we describe the simulation procedure.

\subsection{The Deterministic Model}

The deterministic model assumes that $\mathrm{S}(\mathrm{t}), \mathrm{I}(\mathrm{t})$ and $\mathrm{A}(\mathrm{t})$ are deterministic functions of time ignoring completely the random nature of these numbers. Assuming a homogenous model, the system of differential equations for the deterministic model based on the assumptions given below are

$$
\begin{gathered}
\frac{d S}{d t}=-S \alpha I / N \\
\frac{d I}{d t}=\frac{S \alpha I}{N}-\left(d_{1}+\gamma\right) I \\
\frac{d A}{d t}=\gamma I-d_{1} A-d_{2} \mathrm{~A}
\end{gathered}
$$

where $\alpha>0, \gamma>0, d_{1} \geq 0, d_{2}>0$.

And $\mathrm{S}+\mathrm{I}+\mathrm{A}=\mathrm{N}$

$\alpha=$ Rate at which a susceptible becomes an infective

$\gamma=$ Rate at which an infective becomes AIDS case

$\mathrm{d}_{2}=$ AIDS death rate

$\mathrm{d}_{1}=$ Natural death rate

$\mathrm{N}=$ Population size.

\subsection{Discrete - Time Markov Process Model}

The stochastic model is formulated in terms of the stochastic process. The stochastic process is a collection of random variables, in this model they are $\mathrm{S}(\mathrm{t}), \mathrm{I}(\mathrm{t})$ and $\mathrm{A}(\mathrm{t})$ denoting random variable for numbers of susceptible, infectives and AIDS people respectively at time t. The time is discrete and the values of the random variables are also discrete state spaces. The future state of the process depends only on the current state.

The stochastic equations based on the deterministic model are.

$$
\begin{gathered}
\mathrm{S}(\mathrm{t}+\Delta \mathrm{t})=\mathrm{S}-\mathrm{SI} \alpha / \mathrm{N} \\
\mathrm{I}(\mathrm{t}+\Delta \mathrm{t})=\mathrm{I}+\mathrm{SI} \alpha / \mathrm{N}-\left(\mathrm{d}_{1}+\gamma\right) \mathrm{I} \\
\mathrm{A}(\mathrm{t}+\Delta \mathrm{t})=\mathrm{A}+\gamma \mathrm{I}-\mathrm{d}_{1} \mathrm{~A}-\mathrm{d}_{2} \mathrm{~A}
\end{gathered}
$$

The random variable $\mathrm{S}$ can be expressed in terms of the other variables, that is, $\mathrm{S}(\mathrm{t})=\mathrm{N}-\mathrm{I}(\mathrm{t})-\mathrm{A}(\mathrm{t})$. The DTMC model is described in terms of the dynamics of the two random variables $\mathrm{I}(\mathrm{t})$ and $\mathrm{A}(\mathrm{t})$. Then $\mathrm{I}(\mathrm{t}), \mathrm{A}(\mathrm{t}) \in\{0,1, \ldots, \mathrm{N}\}$ and $\mathrm{I}(\mathrm{t})+\mathrm{A}(\mathrm{t}) \leq \mathrm{N}$. These two variables have a joint probability function.

$\mathrm{P}(\mathrm{i}, \mathrm{a})(\mathrm{t})=\operatorname{Prob}\{\mathrm{I}(\mathrm{t})=\mathrm{i}, \mathrm{A}(\mathrm{t})=\mathrm{a}\}$.

Let $\Delta \mathrm{I}$ denote change in the random variable $\mathrm{I}$ at the time interval $(\mathrm{t}, \mathrm{t}+\Delta \mathrm{t})$.

Let $\Delta \mathrm{A}$ denote change in the random variable $\mathrm{A}$ at the time interval $(\mathrm{t}, \mathrm{t}+\Delta \mathrm{t})$

The transition probabilities for the multivariate markov process are given as follows:

$\mathrm{P}(\mathrm{i}+\mathrm{j}, \mathrm{a}+\mathrm{k}),(\mathrm{i}, \mathrm{a}) \Delta \mathrm{t}=\operatorname{Prob}\{(\Delta \mathrm{I}, \Delta \mathrm{A})=(\mathrm{j}, \mathrm{k}) /(\mathrm{I}(\mathrm{t}), \mathrm{A}(\mathrm{t})=$ $(\mathrm{i}, \mathrm{a})\}$

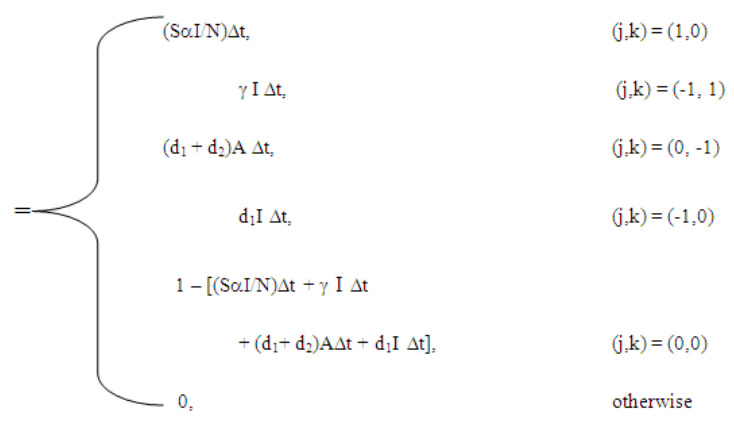

Where,

$\mathrm{j}=0$ implies that no change in the number of infectives,

$\mathrm{j}=1$ implies an increase in the number of infectives by one,

$\mathrm{j}=-1$ implies a decrease in the number of infectives by one,

$\mathrm{k}=0$ implies no change in the number of AIDS,

$\mathrm{k}=1$ implies an increase in the number of AIDS by one

$\mathrm{k}=-1$ implies a decrease in the number of AIDS by one.

The process is time homogenous.

Using the lower case letters $\mathrm{s}$, $\mathrm{i}$ and a to denote the values of the random variables $\mathrm{S}, \mathrm{I}$ and $\mathrm{A}$, the joint probability function,

$\mathrm{P}(\mathrm{i}, \mathrm{a})(\mathrm{t})=\operatorname{Prob}\{(\mathrm{t})=\mathrm{i}, \mathrm{A}(\mathrm{t})=\mathrm{a}\}$ is the solution of the forward Kolmogrov differential equations.

$$
\frac{d p(i, a)}{D t}=\frac{\alpha(s+1)(i-1)}{N}_{P(i-1, a)}+\gamma(i+1)_{P(i+1, a-1)}+\left(d,+d_{2}\right)(a+1)_{P(i, a+1)}+d_{1}(i+1)_{P(i+1, a)}-\left[\alpha s i / N+\gamma i+\left(d_{1}+d_{2}\right) a+d_{1} i\right]_{P(i, a)}
$$

Since our goal is to study the behaviour when $I \neq 0$, we use numerical simulations based on the transition probabilities (instead of applying the forward kolmogrov equations) to study the behaviour of the stochastic model.

\subsection{Assumptions of the Model}

Consider a large population of human adults consisting of males and females. Then there are three kinds of people 
relevant to HIV epidemic in the population. These are the S people (Susceptible people), I people (infective people) and A people (AIDS cases). S people are without the virus but can contract it to become I people through sexual contact with I people or A people or by sharing needles intravenous drug use or through blood transfusion of contaminated blood. I people are those who have contracted the virus and can transmit to $\mathrm{S}$ people through sexual contact or sharing contaminated needles with I people. According to CDC, (1992) quoted by Tan and Xiang (1999), an I person will be classified as a clinical AIDS patient (A person) when this person develops AIDS symptoms or who has $\mathrm{CD}^{+} \mathrm{T}-$ cell counts fall below $200 / \mathrm{mm}^{3}$.

The following assumptions are made by Tan Wai (1999).

(a) Because of the awareness of AIDS, there are no sexual contact or intravenous drug contact with AIDS cases.

(b) There is only one sexual activity level so that each person in the population has the same number of different sexual partners per unit time.

(c) AIDS spread through sexual contact mainly between $\mathrm{S}$ people and I people, ignoring other transmission avenues.

(d) The number of immigrants is equal to the number of deaths and migration out of the $\mathrm{S}$ people and I people in the population.

(e) The infection duration of the I people have no significant impacts on the HIV transmission. Then let $\gamma \Delta \mathrm{t}$ be the probability of I person becoming A person during $(\mathrm{t}, \mathrm{t}+\Delta \mathrm{t})$.

(f) People pick up their sexual partners randomly from the population (random mixing or proportional mixing).

Let $\mathrm{N}(\mathrm{t})=\mathrm{S}(\mathrm{t})+\mathrm{I}(\mathrm{t})$ and let $\alpha \mathrm{I} / \mathrm{N} \Delta \mathrm{t}$ be

The probability of $\mathrm{S}$ individual becoming an I individual.

\subsection{Simulation Procedure}

Matlab program is used to simulate the discrete time markov chain epidemic model. $\mathrm{S}(\mathrm{t}), \mathrm{I}(\mathrm{t})$ and $\mathrm{A}(\mathrm{t})$ denoting the susceptible, the infective and the AIDS cases which are the random variables such that $\mathrm{S}(\mathrm{t}), \mathrm{I}(\mathrm{t})$ and $\mathrm{A}(\mathrm{t}) \in\{\mathrm{O}$, $\mathrm{I}, \ldots, \mathrm{N}\}$, and $\mathrm{N}=\mathrm{S}(\mathrm{t})+\mathrm{I}(\mathrm{t})+\mathrm{A}(\mathrm{t})$. The outcome of $\mathrm{S}(\mathrm{t}), \mathrm{I}(\mathrm{t})$ and $\mathrm{A}(\mathrm{t})$ depend on the transition probabilities. The initial conditions are $\mathrm{N}=100, \mathrm{I}(0)=2$ and $\mathrm{A}(0)=0$

Given the set of parameters; rate at which susceptible becomes infective $(\alpha)$, rate at which infective becomes AIDS $(\gamma)$, AIDS death rate $\left(d_{2}\right)$ and natural death rate $\left(d_{1}\right)$, the simulation was done such that an epidemic would develop. The value of each parameter is varied while values of other parameters are fixed.

(a) When $\alpha$ is varied $(0.08,0.1,0.3,0.5,0.8)$ and other parameters are fixed at $\gamma=0.0245, d_{1}=$ $0.0045, d_{2}=0.0012$.

(b) When $\gamma$ is varied $(0.00045,0.0045,0.0145,0.0445)$ while other parameters are fixed at $\alpha=0.3, d_{1}=$ $0.0012, d_{2}=0.0045$.

(c) When $\mathrm{d}_{2}$ is varied $(0.00085,0.0015,0.0045,0.0105)$ while other parameters are fixed at $\alpha=0.3, \gamma=$
$0.0245, d_{1}=0.0012$.

(d) When $d_{1}$ is varied $(0.00012,0.0012,0.0032,0.0062$, 0.0102 ) while other parameters are fixed at $\alpha=$ $0.3, \gamma=0.0245, d_{1}=0.0045$

\section{Results}

The results from the simulated data were obtained and presented in graphs. The pattern of the three disease states were studied with respect to time. The effects of the parameters $\gamma, d_{1}$ and $d_{2}$ are studied on the infectives and AIDS cases, while the effect of $\alpha$ is studied on the three disease states.

\subsection{Stochastic Behaviour of the Three Disease States}

The stochastic behaviour of the three disease states is shown in Figure 3.1, where rate at which susceptible becomes infective $(\alpha)$ is 0.29 , rate at which infective becomes AIDS $(\gamma)$ is 0.0245 , natural death rate $\left(\mathrm{d}_{1}\right)$ is 0.0012 and AIDS death rate $\left(\mathrm{d}_{2}\right)$ is 0.0045 , and the population size $\mathrm{N}$ is 100 . The figure typifies what occurs after the introduction of $2 \%$ infectives in a population of susceptibles at the given parameter values. An epidemic is observed as a wave of susceptibles decay. The susceptible curve shows a negative exponential depletion of the susceptibles, decreasing with a decreasing rate as time increases. The number of infectives increases rapidly until most susceptibles are infected. Number of susceptibles decreases at the same rate as infectives increases, and the rate of change has an effect on the rate at which AIDS cases appear. The curve of the infectives is of the log logistic curve. The curve starting from $2 \%$ infectives increases at a decreasing rate as time increases to a peak of $62 \%$ infectives, thereafter decreases with an increasing rate as time increases. The susceptibles curve and the AIDS curve intersect at a time when the infectives curve is at its peak. At this point the susceptibles have decayed to $19 \%$ while the AIDS cases have increased to $19 \%$.

\subsection{Effect of Changes in Rates at which Susceptible Becomes Infective $(\alpha)$}

Keeping the parameters $\gamma, \mathrm{d}_{1}$ and $\mathrm{d}_{2}$ constants at the values $0.0245,0.0012$ and 0.0045 respectively, the effect of changing $\alpha$ on the susceptibles is shown in Figure 3.2. When $\alpha$ is low $(\leq 1 \%)$, the number of susceptibles reduces rapidly until there are about $67 \%$, and thereafter remains constant. When $\alpha$ is high $(>1 \%)$, the number of susceptibles reduces more rapidly until no more susceptibles. The rate of decrease in the number of susceptibles increases with increases in $\alpha$. The effect of changing $\alpha$ on the infectives is shown in Figure 3.3. When $\alpha$ is low $(\leq 1 \%)$, the number of infective increases rapidly to a peak of $23 \%$ infectives, and thereafter remains constant. When $\alpha$ is high $(\geq 1 \%)$, the number of infectives increases more rapidly to higher peaks of higher percentages of infectives, and thereafter the number of infectives decreases 
with increase in time. The rate of increase in the number of infectives increases with increase in $\alpha$ and the rate of decrease after the peaks, increases with increase in $\alpha$. All the infectives curves showed fluctuations. The more the fluctuations, the lesser the value of $\alpha$. The effect of changing $\alpha$, on the AIDS cases is shown in Figure 3.4. When $\alpha$ is low $(\leq 1 \%)$, the number of AIDS cases increases to $10 \%$, and thereafter remains constant. When $\alpha$ is high $(>1 \%)$, the number of AIDS cases increases slowly at the beginning and later towards the end increases rapidly to higher percentages of AIDS cases. The rate of increase in the number of AIDS cases is more slow at the beginning and more rapid towards the end with increase in $\alpha$.

\subsection{Effect of Changes in Rate at which Infective Becomes $\operatorname{AIDS}(\gamma)$}

Keeping the parameter $\alpha, \mathrm{d}_{1}$ and $\mathrm{d}_{2}$ constant at the values $0.3,0.0012$ and 0.0045 respectively, the effect of changing $\gamma$ on the infectives is shown in Figure 3.5. When $\gamma$ is low $(\leq 0.45 \%)$, the number of infectives increases rapidly to a peak of $84 \%$ infectives, and thereafter decreases a little and remains constant. The lower the value of $\gamma$, the higher the peak of infectives and the earlier in time it remains constant When $\gamma$ is high $(>0.45 \%)$, the number of infectives increases less rapidly to lesser peaks, and thereafter decreases. The higher the value of $\gamma$, the less the rate of increase of the number of infectives and lower peaks. After the peaks, the rate of decrease in the number of infectives increases with increase in $\gamma$. All the infective curves showed fluctuations. The more the fluctuations, the higher the value of $\gamma$. The effect on changing $\gamma$ on the AIDS cases is shown in Figure 3.6 When $\gamma$ is low $(\leq 0.45 \%)$, the number of AIDS cases increases slowly at the beginning and rapidly towards the end to a maximum of $27 \%$. When $\gamma$ is higher $(>0.45 \%)$, the number of AIDS cases increases slowly at the beginning and more rapidly towards the end to a more higher percentages. The rate of increase in the

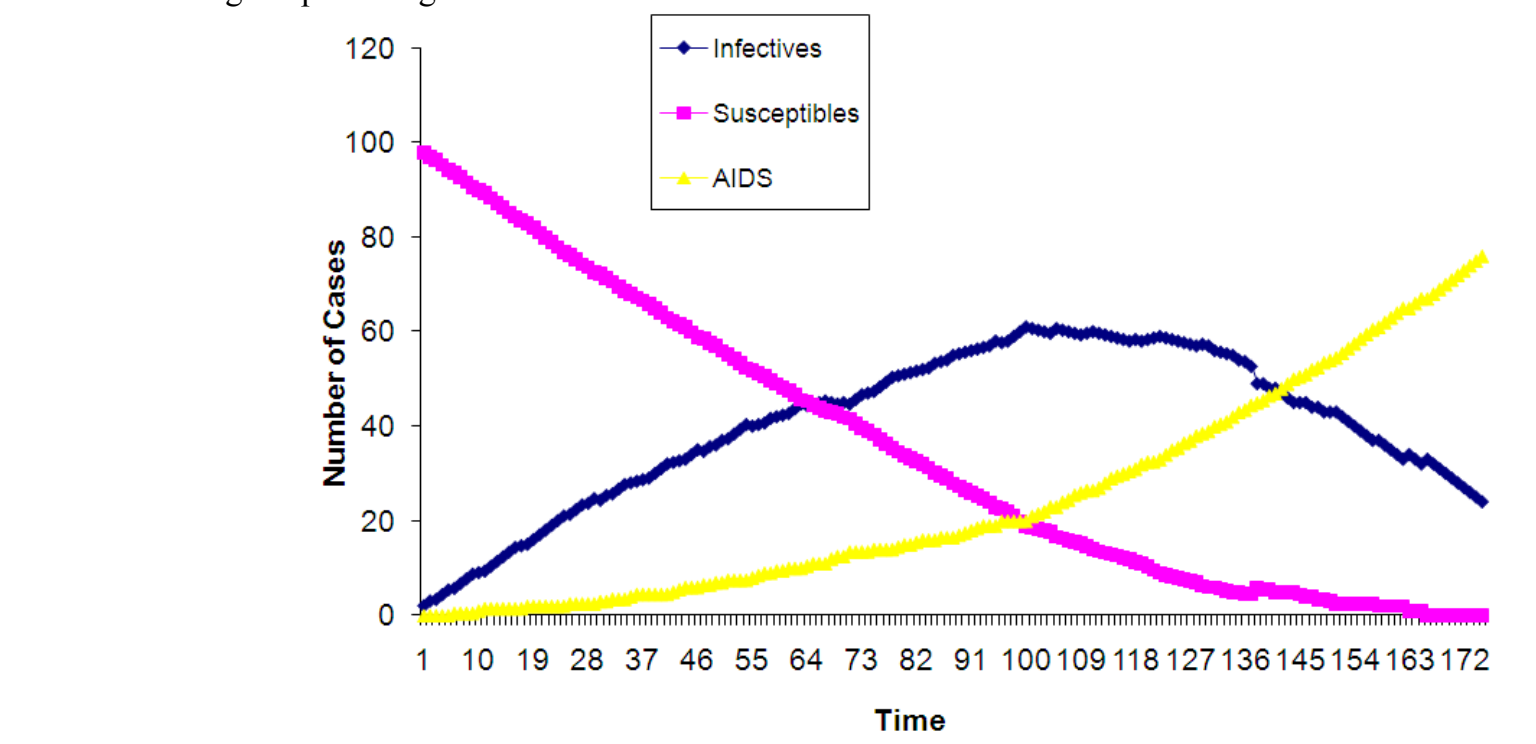

Fig 4.1. Stochastic Behaviour of the Three Disease States with Time for $\alpha=0.29, \gamma=0.0245, d 2=0.0045, \boldsymbol{d} \mathbf{1}=\mathbf{0 . 0 0 1 2}$ number of AIDS at the beginning is decreasingly slow with increase in $\gamma$. And towards the end, the rate of increase in the number of AIDS is increasingly fast with increase in $\gamma$.

\subsection{Effect of Changes in AIDS Death Rates (d $\left.d_{2}\right)$}

Keeping the parameter $\alpha, \gamma$ and $\mathrm{d}_{1}$ constants at the values $0.3,0.0245$ and 0.0012 respectively, the effect of changing AIDS death rate $\left(\mathrm{d}_{2}\right)$ on the infectives is shown in Figure 3.7. At the beginning, all the curves show about the same rate of increase in the number of infectives. But towards the peaks, the rate of increase in the number of infectives increases with decrease in $\mathrm{d}_{2}$. After the peaks, the curves decrease, with the higher level of $\mathrm{d}_{2}$ decreasing more. The curves show so much fluctuations. The effect on changing $\mathrm{d}_{2}$ on the AIDS cases is shown in Figure 3.8. At the beginning, all the curves show about the same slow increase in the number of AIDS cases. Towards the end, the rate of increase in the number of AIDS cases is faster and a little higher with increase in the level of $\mathrm{d}_{2}$.

\subsection{Effect of Changes in Natural Death Rates $\left(d_{1}\right)$}

Keeping the parameter $\alpha, \gamma$ and $\mathrm{d}_{2}$ constant at the values $0.3,0.0245$ and 0.0045 respectively, the effect of changing $\mathrm{d}_{1}$ on the infectives is shown in Figure 4.9. At the beginning, all the curves show about the same rate of increase in the number of infectives. But towards the peaks, the rate of increase in the number of infectives increases little with decrease in the level of $d_{1}$. After the peaks, the curves decrease more with increase in the level of $d_{1}$. The curves show so much fluctuations. The effect of changing $\mathrm{d}_{1}$ on the AIDS cases is shown in Figure 4.10. At the beginning, all the curves showed about the same slow increase in the number of AIDS cases. Towards the end, the rate of increase in the number of AIDS cases is faster and little higher with increase in the level of $\mathrm{d}_{1}$. 


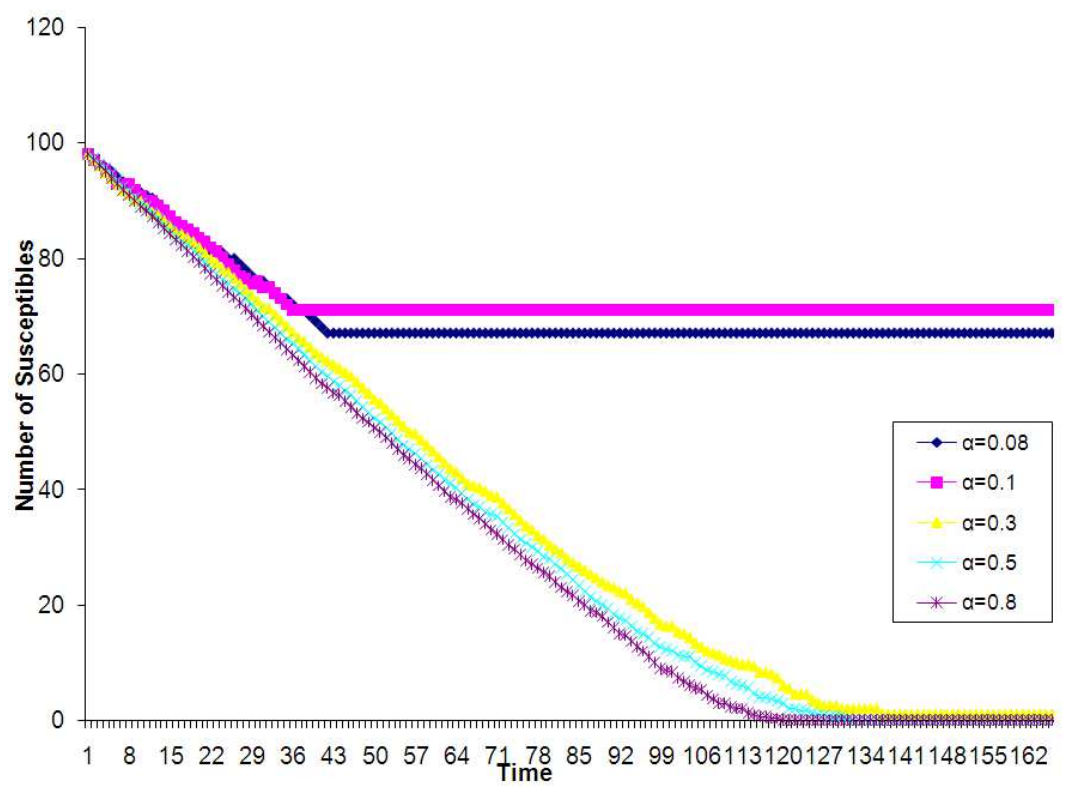

Fig 4.2. Changes in Number of Susceptibles with Changes at Rates Susceptibles Becomes Infectives

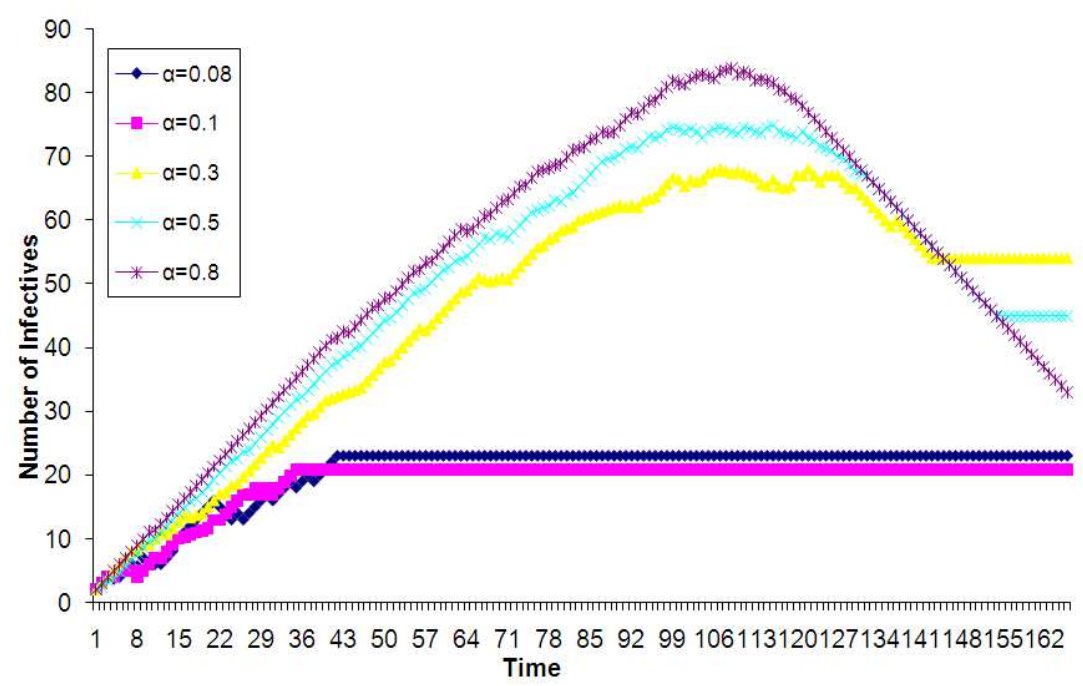

Fig 4.3. Changes in Number of Infectives with Changes at Rates Susceptible Becomes Infective

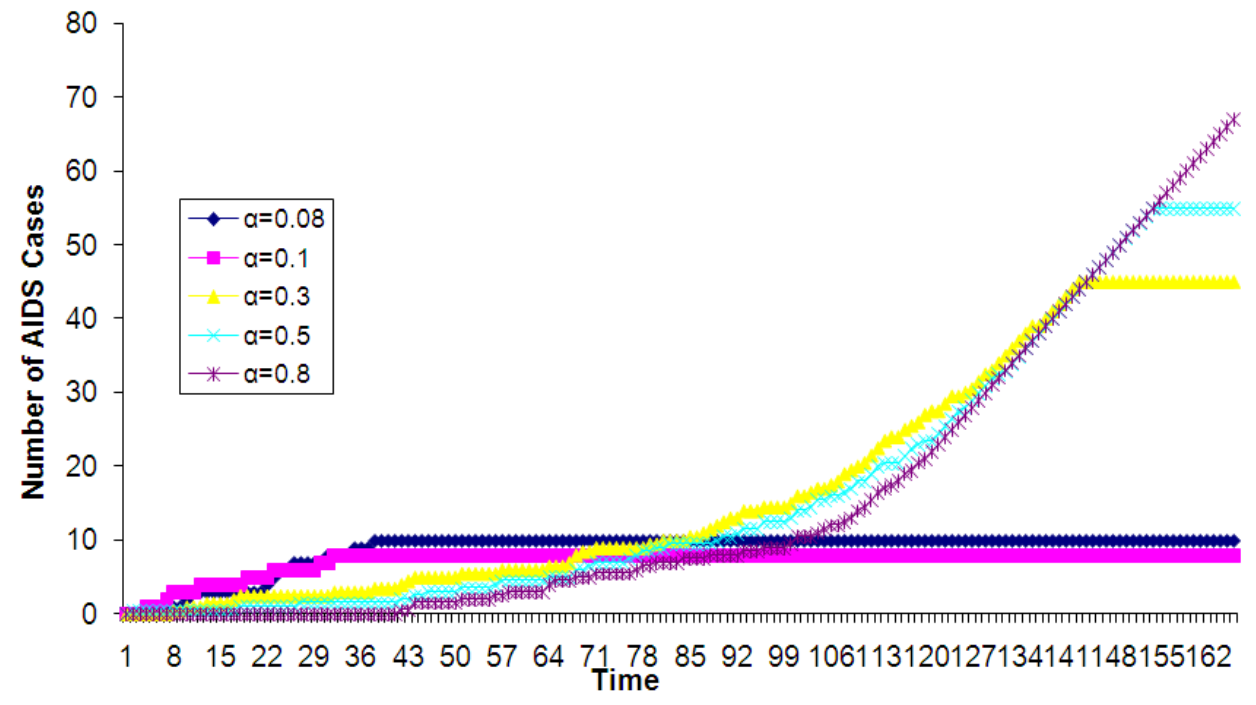

Fig 4.4. Changes in Number of AIDS Cases with Changes at Rates Susceptible becomes Infective 


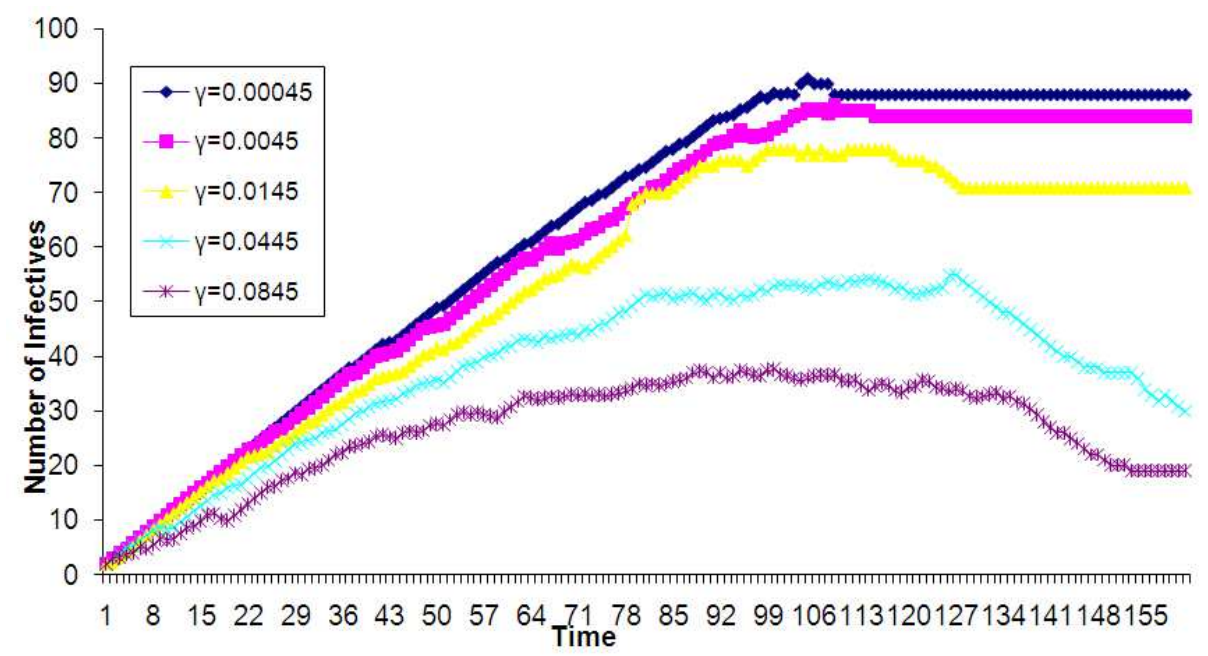

Fig 4.5. Changes in Number of Infectives with Changes at Rates Infective Becomes AIDS

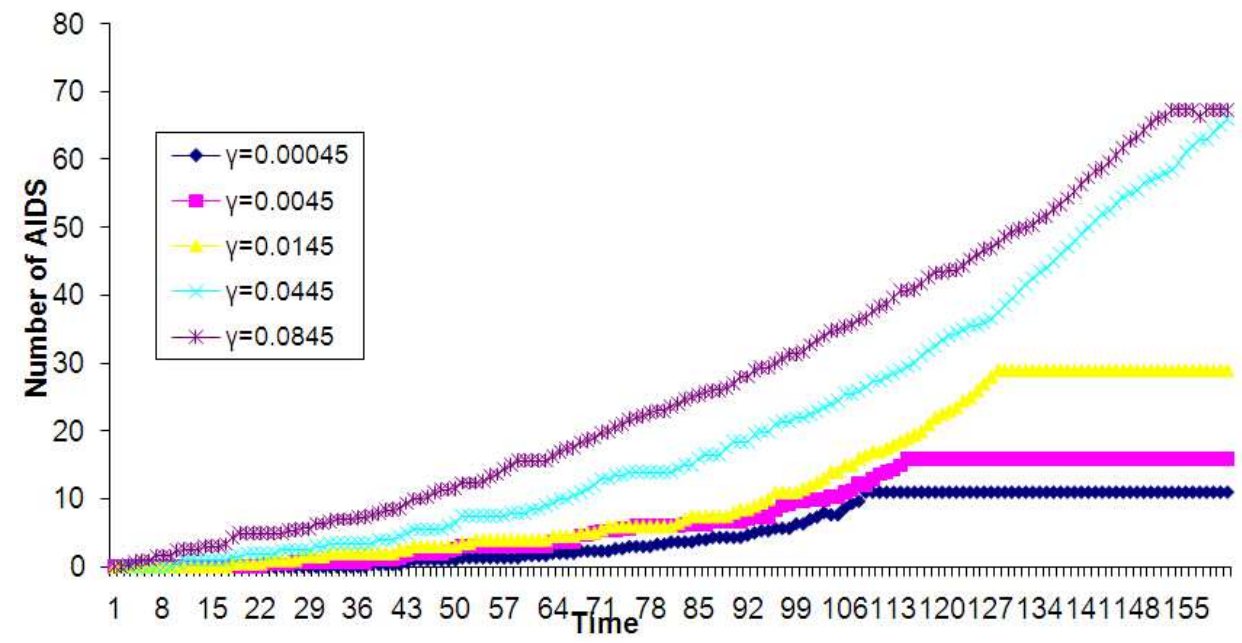

Fig 4.6. Changes in Number of AIDS Cases with Changes at Rates Infective Becomes AIDS

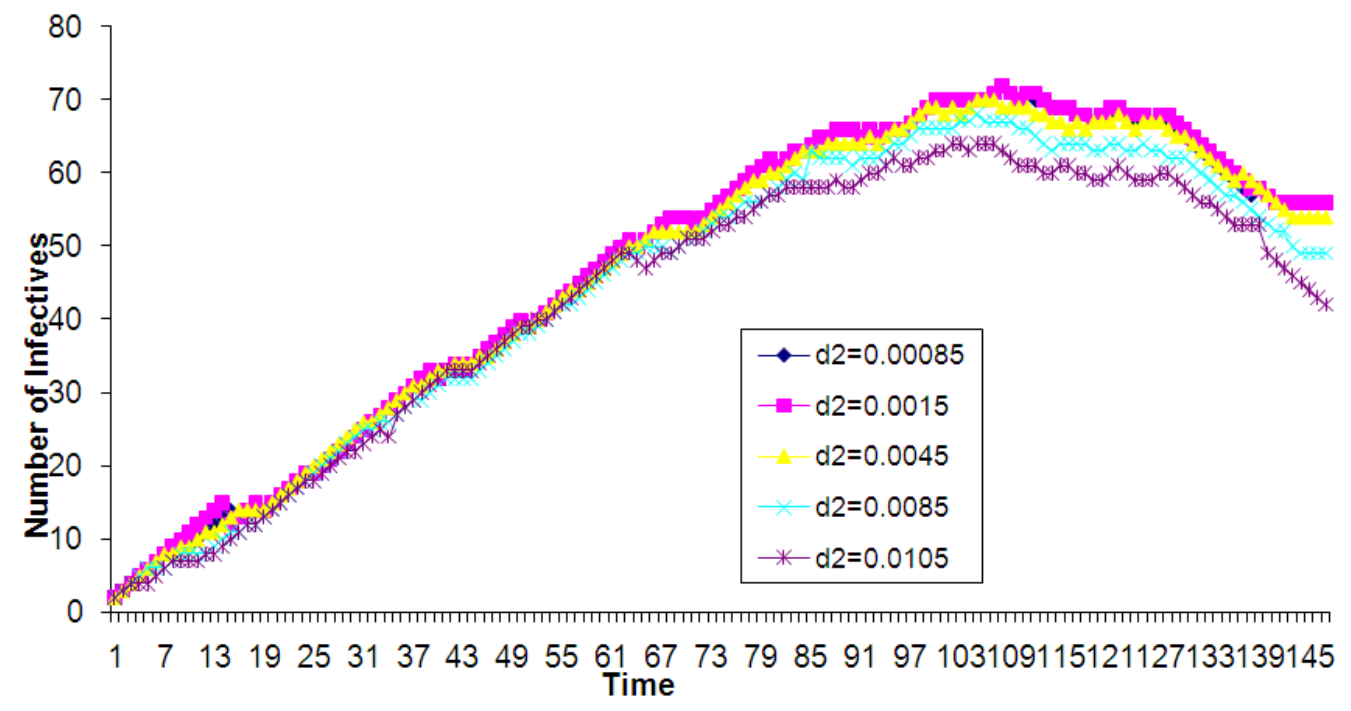

Fig 4.7. Changes in Number of Infectives with Changes in AIDS Death Rates 


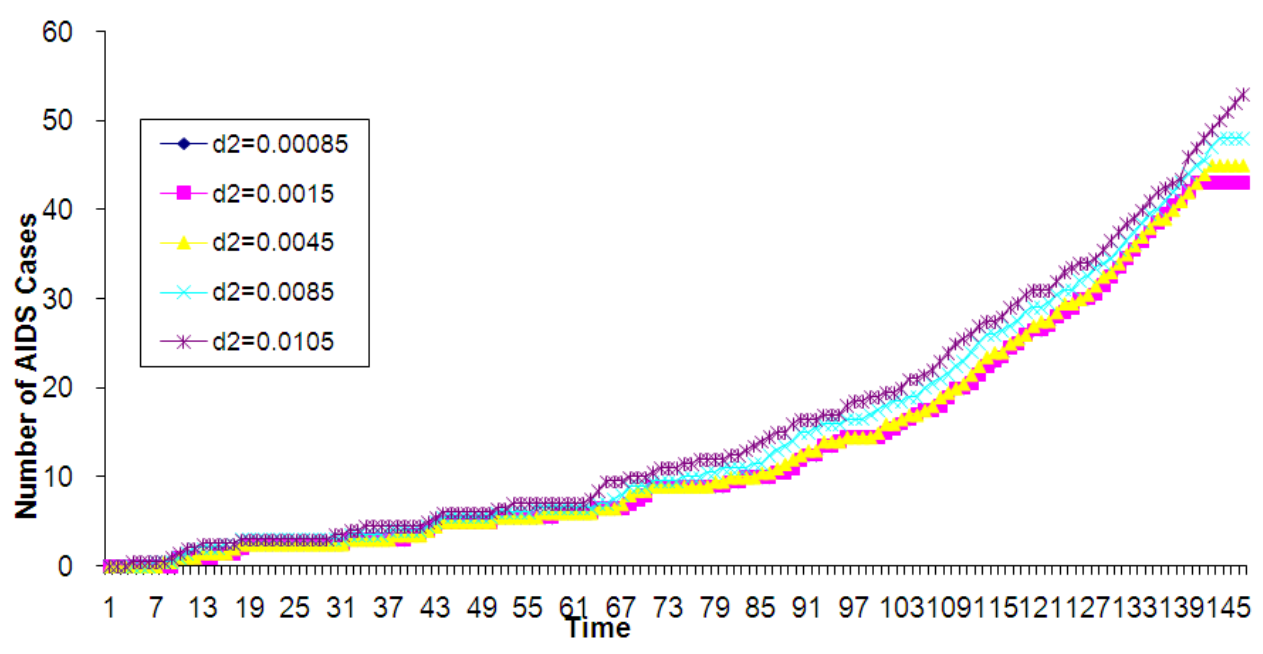

Fig 4.8. Changes in Number of AIDS Cases with Changes in AIDS Death Rates

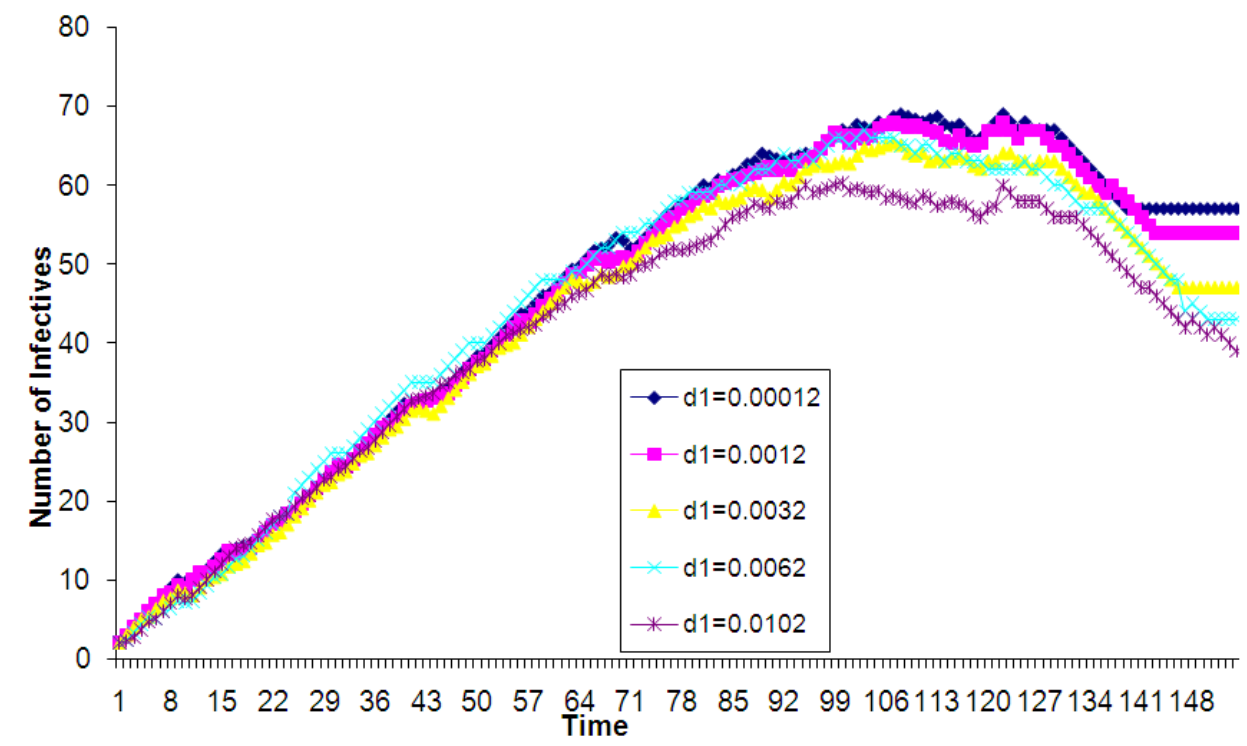

Fig 4.9. Changes in Number of Infectives with Changes in Natural Death Rates

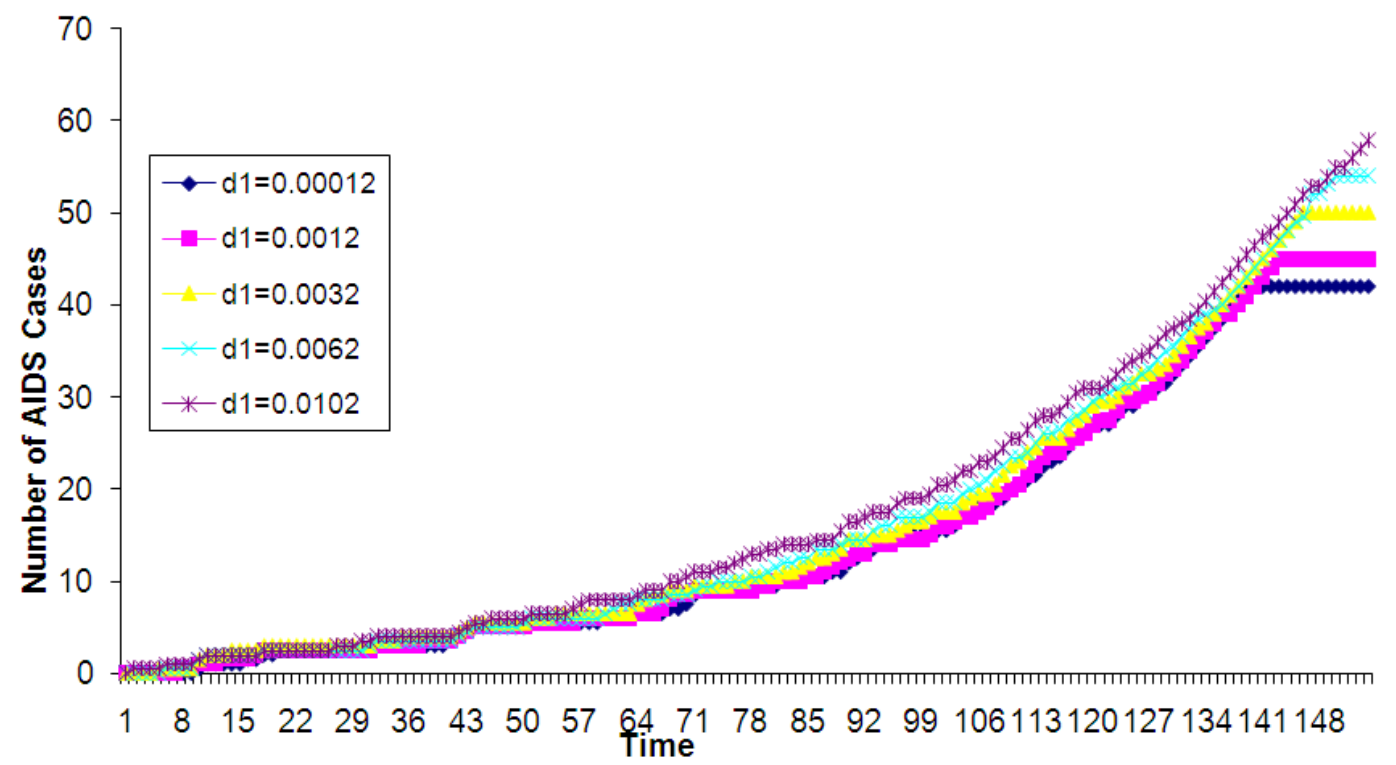

Fig 4.10. Changes in Number of AIDS Cases with Changes at Natural Death Rates 


\section{Discussion and Recommendation}

The assumption that the susceptible people contract HIV disease only through sexual contact with the infective people is realistic. This is because sexual contact between the susceptible people and the infective people is the commonest way for susceptible people contracting HIV disease in Nigeria. Other modes of transmission are not as common. The Discrete - Time Markov process HIV/AIDS model is able to show the epidemic of the HIV/AIDS disease. The number of susceptibles shows depletion in a negative exponential form, and the number of infectives shows growing and decaying in a log logistic form, while the number of AIDS shows growth in a positive exponential form. Changing the levels of the parameters in the model give insight into the influences of these parameters on the number of these three disease states. From the effects of these parameters on the three disease states, the crucial parameters to control HIV/AIDS are determined. When the rate at which susceptible becomes infective $(\alpha)$ is not more than $1 \%$ ensures the depletion of the number of susceptibles stop early with a reasonably large size. The numbers of infectives and AIDS cases are kept to a minimum and the numbers stabilize faster. Therefore to control the epidemic $\alpha$ should be kept very low. To control the epidemic, rate at which infective becomes AIDS $(\gamma)$ need to be kept lower than $0.45 \%$. When $\gamma$ is lower than $0.45 \%$ the number of infectives is higher but the number of AIDS cases is lower. When AIDS death rate is low, number of AIDS cases is reduced while the number of infectives is higher. The situation is the same for natural death rate as that of the AIDS death rate.

To keep the numbers of infectives and AIDS cases low, it is necessary for the rate at which susceptible becomes infective $(\alpha)$ be made lower than $1 \%$ and the rate infective becomes AIDS $(\gamma)$ to be lower than $0.45 \%$.

\section{References}

[1] Coffin, J. et al. (1986). Human Immunodeficiency Viruses. World Scientific Publishing Company Limited.

[2] Druten Van, J. A. M., DeBoo, Th., Reintjes, A.g.M., Jager, J. C., Heisterkamp, S. H., Continho, R. A., Bos, J. M. and Ruitenberg, E. J. (1987). Reconstruction and prediction of spread of HIV infection in populations of homosexual men.

[3] Isham Valerie (1988). Mathematical Modelling of the Transmission Dynamics of HIV Infection and AIDS.

[4] Londa J. S. Allen and Amy M. Burgin (1998) Comparism of deterministic and stochastic SIS and SIR models in discrete time.

[5] Nasidi A, Henry T.O., Ajose Coker O.O., and et al. Evidence of LAV/HTLV III Infection and AIDS related complex in Lagos, Nigeria.

[6] Tan, W. Y. and Xing, Z. H. (1999). Modeling the HIV epidemic with variable infection in homosexual populations by state space model. 